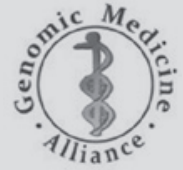

Genomic Medicine Alliance (GMA)

European Alliance for Personalised Medicine

\title{
Editor-in-Chief
}

N. Probst-Hensch, Basel, Switzerland

\section{Scientific Editors}

M.J. Khoury, Atlanta, Ga., USA

S. Wallace, Leicester, UK

\section{Editorial Board}

R. Adany, Debrecen, Hungary

A. Aaro, Odense, Denmark

D. Avard, Montreal, Que., Canada

I. Blancquaert, Montreal, Que., Canada

J.-J. Cassiman, Leuven, Belgium

E.E. Castilla, Rio de Janeiro, Brazil

S. Grosse, Atlanta, Ga., USA

J. Harris, Oslo, Norway

A. Haslberger, Vienna, Austria

M. Karmali, Toronto, Ont., Canada

H. Lehrach, Berlin, Germany

J. Little, Ottawa, Ont., Canada

N. Malats, Madrid, Spain

C. McBride, Atlanta, Ga., USA

\section{Associate Editors}

G.P. Patrinos, Patras, Greece
P. O'Leary, Perth, W.A., Australia

F. Paccaud, Epalinges, Switzerland

B. Peterlin, Ljubljana, Slovenia

C.N. Rotimi, Bethesda, Md., USA

P. Schröder-Bäck, Maastricht,

The Netherlands

D. Taruscio, Rome, Italy

H. van Kranen, Bilthoven,

The Netherlands

B. van Ommen, Zeist,

The Netherlands

$\mathrm{H}$. van Oyen, Brussels, Belgium

$\mathrm{H}$. Vondeling, Odense, Denmark

J. Wilkinson, Stockton, UK

H. Yang, Shenzhen, China 
S. Karger

Medical and Scientific Publishers

Basel $\cdot$ Freiburg $\cdot$ Paris $\bullet$ London .

New York $\cdot$ Chennai $\cdot$ New Delhi $•$

Bangkok $\cdot$ Beijing $\cdot$ Shanghai $\cdot$ Tokyo $\cdot$

Kuala Lumpur $\cdot$ Singapore $\cdot$ Sydney

\section{Disclaimer}

The statements, opinions and data contained in this publication are solely those of the individual authors and contributors and not of the publisher and the editor(s). The appearance of advertisements in the journal is not a warranty, endorsement, or approval of the products or services advertised or of their effectiveness, quality or safety. The publisher and the editor(s) disclaim responsibility for any injury to persons or property resulting from any ideas, methods, instructions or products referred to in the content or advertisements.

Drug Dosage

The authors and the publisher have exerted every effort to ensure that drug selection and dosage set forth in this text are in accord with current recommendations and practice at the time of publication. However, in view of ongoing research, changes in government regulations, and the constant flow of information relating to drug therapy and drug reactions, the reader is urged to check the package insert for each drug for any change in indications and dosage and for added warnings and precautions. This is particularly important when the recommended agent is a new and/or infrequently employed drug.
All rights reserved

No part of this publication may be translated into other languages, reproduced or utilized in any form or by any means, electronic or mechanical, including photocopying, recording, microcopying, or by any information storage and retrieval system, without permission in writing from the publisher or in the case of photocopying, direct payment of a specified fee to the Copyright Clearance Center (see 'General Information')

(c) Copyright 2017 by S. Karger AG

CH-4009 Basel (Switzerland)

Printed on acid-free and non-aging paper (ISO 9706)

\section{KARGER}




\section{Public Health Genomics}

No. 1

Reviews

1 Reimbursed Price of Orphan Drugs: Current Strategies and Potential Improvements

Mincarone, P. (Brindisi); Leo, C.G. (Lecce/Boston, MA); Sabina, S. (Lecce); Sarriá-Santamera, A. (Madrid); Taruscio, D. (Rome); Serrano-Aguilar, P.G. (Madrid/Santa Cruz de Tenerife); Kanavos, P. (London)

9 Genomics of Cardiometabolic Disorders in Sub-Saharan Africa Adebamowo, S.N. (Baltimore, MD); Tekola-Ayele, F.; Adeyemo, A.A.; Rotimi, C.N. (Bethesda, MD)

Original Papers

27 Research Ethics 2.0: New Perspectives on Norms, Values, and Integrity in Genomic Research in Times of Even Scarcer Resources

Brall, C. (Maastricht/Bern); Maeckelberghe, E. (Groningen); Porz, R. (Bern); Makhoul, J. (Beirut); Schröder-Bäck, P. (Maastricht/Bremen)

36 Direct-to-Consumer Genetic Testing: User Motivations, Decision Making, and Perceived Utility of Results Roberts, J.S.; Gornick, M.C. (Ann Arbor, MI); Carere, D.A. (Hamilton, ON); Uhlmann, W.R. (Ann Arbor, MI); Ruffin, M.T. (Hershey, PA); Green, R.C. (Boston, MA)

46 Community Perceptions of Biobanking Participation: A Qualitative Study among Mexican-Americans in Three Texas Cities

Heredia, N.I.; Krasny, S.; Strong, L.L. (Houston, TX); Von Hatten, L. (Dallas, TX); Nguyen, L. (Houston, TX); Reininger, B.M. (Brownsville, TX); McNeill, L.H.; Fernández, M.E. (Houston, TX)

Short Communication

58 Personalised Medicine: A New Approach to Improving Health in Indigenous Australian Populations

Rae, K.M. (Tamworth, NSW/New Lambton, NSW); Grimson, S.

(New Lambton, NSW); Pringle, K.G. (New Lambton, NSW/

Callaghan, NSW)

\section{No. 2}

Cancer Genomics and Public Health

Guest Editors: Malats, N.; (Madrid); Katsila, T. (Patras); Patrinos, G.P. (Patras)

Editorial

67 Cancer Genomics and Public Health

Malats, N. (Madrid); Katsila, T. (Patras); Patrinos, G.P. (Patras/Al Ain)
Reviews

70 Cancer Precision Medicine: Why More Is More and DNA Is Not Enough

Schütte, M.; Ogilvie, L.A.; Rieke, D.T.; Lange, B.M.H.; Yaspo, M.-L.; Lehrach, H. (Berlin)

81 Pancreas Cancer Precision Treatment Using Avatar Mice from a Bioinformatics Perspective

Perales-Patón, J.; Piñeiro-Yañez, E.; Tejero, H.; López-Casas, P.P. (Madrid); Hidalgo, M. (Boston, MA); Gómez-López, G.; Al-Shahrour, F. (Madrid)

92 Genomics in Primary and Secondary Prevention of Pancreatic Cancer

Malats, N.; Molina-Montes, E. (Madrid); La Vecchia, C. (Milan)

100 Epigenomics, Pharmacoepigenomics, and Personalized Medicine in Cervical Cance

Kabekkodu, S.P.; Chakrabarty, S.; Ghosh, S. (Karnataka); Brand, A. (Maastricht); Satyamoorthy, K. (Karnataka)

116 Exosomes: A Cancer Theranostics Road Map

Panagiotara, A. (Patras); Markou, A.; Lianidou, E.S. (Athens); Patrinos, G.P. (Patras/Al Ain); Katsila, T. (Patras)

Original Papers

126 DoriTool: A Bioinformatics Integrative Tool for Post-Association Functional Annotation

Martín-Antoniano, I.; Alonso, L.; Madrid, M.; López de Maturana, E. Malats, N. (Madrid)

136 1-CMDb: A Curated Database of Genomic Variations of the One-Carbon Metabolism Pathway

Bhat, M.K.; Gadekar, V.P.; Jain, A.; Paul, B.; Rai, P.S.; Satyamoorthy, K. (Manipal)

142 Application of the DruGeVar Database in Cancer Genomics and Pharmacogenomics

Sarris, K. (Patras); Komianou, A. (London); Patrinos, G.P. (Patras/Al Ain); Katsila, T. (Patras)

148 Author Index/Subject Index

No. 3

Original Papers

149 Precision Genetic and Genomic Medicine in the Middle East and North Africa Region: Are We There Yet? Bilani, N.; Dagher, M.; Zgheib, N.K. (Beirut)

158 Subjects at Risk for Genetic Late-Onset Neurological Diseases: Objective Knowledge Leite, Â. (Lisbon/Porto); Leite, F.; Dinis, M.A.P. (Porto)

166 Assessment of Direct-to-Consumer Genetic Testing Policy in Korea Based on Consumer Preference

Jeong, G. (Sejong-si)

\section{KARGER}

E-Mail karger@karger.com www.karger.com
(C) 2018 S. Karger AG, Basel

Access to full text and tables of contents, including tentative ones for forthcoming issues: www.karger.com/phg_issues 
174 Exploring the Use of a Participative Design in the Early Development of a Predictive Test: The Importance of Physician Involvement

Veilleux, S.; Villeneuve, M.; Lachapelle, N. (Québec, QC); Kohen, R.; Vachon, L.; White Guay, B.; Rioux, J.D.; Bitton, A. (Montréal, QC) for the IBD Genomic Medicine Consortium (iGenoMed)

188 A Common Ancestral Asn242Ser Mutation in TMEM67 Identified in Multiple Iranian Families with Joubert Syndrome Dehghani, M. (Yazd); Mojarad, M.; Ghayoor Karimiani, E. (Mashhad); Vahidi Mehrjardi, M.Y. (Yazd); Sahebalzamani, A. (Kerman); Ashrafzadeh, F; Beiraghi Toosi, M.; Eslahi, A.; Ahangari, N. (Mashhad); Yassini, S.M.; Hassanbeigi, A.; Rasti, A.; Kalantar, S.M. (Yazd); Maroofian, R. (Exeter)

Short Communication

194 Cancer Genomic Resources and Present Needs in the Latin American Region

Torres, Á. (Bogotá); Oliver, J.; Frecha, C. (Buenos Aires); Montealegre, A.L. (Bogotá); Quezada-Urbán, R.; Díaz-Velásquez, C.E.; Vaca-Paniagua, F. (Mexico City); Perdomo, S. (Bogotá)

No. 4

Review

203 Health-Related Genetic Direct-to-Consumer Tests in the German Setting: The Available Offer and the Potential Implications for a Solidarily Financed Health-Care System Plöthner, M.; Klora, M.; Rudolph, D.; Graf von der Schulenburg, J.-M. (Hannover)

Original Papers

218 Providing Personalised Nutrition: Consumers' Trust and Preferences Regarding Sources of Information, Service Providers and Regulators, and Communication Channels Poínhos, R.; Oliveira, B.M.P.M. (Porto); van der Lans, I.A.; Fischer, A.R.H.; Berezowska, A. (Wageningen); Rankin, A. (Belfast); Kuznesof, S. (Newcastle upon Tyne); Stewart-Knox, B. (Bradford); Frewer, L.J. (Newcastle upon Tyne); de Almeida, M.D.V. (Porto)

229 Prevalence Estimates of Rare Congenital Anomalies by Integrating Two Population-Based Registries in Tuscany, Italy Coi, A.; Santoro, M.; Pierini, A.; Marrucci, S.; Pieroni, F.; Bianchi, F. (Pisa)

235 Online Education and e-Consent for GeneScreen, a Preventive Genomic Screening Study

Cadigan, R.J.; Butterfield, R. (Chapel Hill, NC); Rini, C. (Hackensack, NJ); Waltz, M.; Kuczynski, K.J. (Chapel Hill, NC); Muessig, K.; Goddard, K.A.B. (Portland, OR); Henderson, G.E. (Chapel Hill, NC)

Policy Statement

247 Genetics and Insurance in Australia: Concerns around a SelfRegulated Industry

Newson, A.J. (Sydney, NSW); Tiller, J.; Keogh, L.A. (Melbourne, VIC); Otlowski, M. (Hobart, TAS); Lacaze, P. (Melbourne, VIC)

\section{No. 5}

Reviews

257 Antibiotics-Induced Obesity: A Mitochondrial Perspective Andrade, M.J.; Jayaprakash, C.; Bhat, S. (Manipal); Evangelatos, N. (Maastricht/Nuremberg); Brand, A. (Maastricht/Manipal); Satyamoorthy, K. (Manipal)
274 Metabolomics in Sepsis and Its Impact on Public Health Evangelatos, N.; Bauer, P. (Nuremberg/Maastricht); Reumann, M. (Maastricht/Rüschlikon); Satyamoorthy, K. (Manipal); Lehrach, H. (Berlin); Brand, A. (Maastricht/Manipal)

286 Effects of Diet on Telomere Length: Systematic Review and Meta-Analysis

Pérez, L.M.; Amaral, M.A.; Mundstock, E.; Barbé-Tuana, F.M.; Guma, F.T.C.R.; Jones, M.H.; Machado, D.C. (Porto Alegre); Sarria, E.E. (Santa Cruz); Marques e Marques, M.; Preto, L.T.; Epifanio, M.; Meinem Garbin, J.G.; Mattiello, R. (Porto Alegre)

Original Paper

293 Attitudes towards Personal Genomics and Sharing of Genetic Data among Older Swiss Adults: A Qualitative Study Mählmann, L. (Basel/Maastricht); Schee gen. Halfmann, S. (Maastricht); von Wyl, A. (Zurich); Brand, A. (Maastricht)

307 Errata

\section{No. 6}

Invited Editorial

309 Data, Technology, and Public Health

Borisch, B. (Geneva); Iloegbu, C.A. (New York, NY); Wells, N. (Geneva)

Original Papers

312 Big Data for Public Health Policy-Making: Policy Empowerment

Mählmann, L. (Maastricht/Basel); Reumann, M. (Maastricht/Rüschlikon); Evangelatos, N. (Maastricht/Nuremberg); Brand, A. (Maastricht)

321 Potentials and Challenges of the Health Data Cooperative Model

van Roessel, I. (Maastricht); Reumann, M. (Maastricht/Zurich); Brand, A. (Maastricht)

332 Examining the Cascade of Participant Attrition in a Genomic Medicine Research Study: Barriers and Facilitators to Achieving Diversity

Moore, E.G. (Durham, NC); Roche, M. (Chapel Hill, NC); Rini, C. (Hackensack, NJ); Corty, E.W. (Tallahassee, FL); Girnary, Z. (Durham, SC); O'Daniel, J.M.; Lin, F.-C.; Corbie-Smith, G.; Evans, J.P.; Henderson, G.E.; Berg, J.S. (Chapel Hill, NC)

Short Communication

343 Assessing Genetic Literacy Awareness and Knowledge Gaps in the US Population: Results from the Health Information National Trends Survey

Krakow, M. (Bethesda, MD); Ratcliff, C.L. (Salt Lake City, UT); Hesse, B.W. (Bethesda, MD); Greenberg-Worisek, A.J. (Rochester, MN) 